\title{
Tear cytokine profile of glaucoma patients treated with preservative- free or preserved latanoprost
}

\author{
Jose M. Martinez-de-la-Casa, PhD a, b, *, Francisco Perez-Bartolome, MD a, \\ Elena Urcelay, $\mathrm{PhD}^{\mathrm{c}}$, Jose L. Santiago, $\mathrm{PhD}^{\mathrm{c}}$, Javier Moreno-Montañes, $\mathrm{PhD}^{\mathrm{d}}$, \\ Pedro Arriola-Villalobos, $\mathrm{PhD}^{\mathrm{a}}$, Jose M. Benitez-del-Castillo, PhD ${ }^{\mathrm{a}, \mathrm{b}}$, \\ Julian Garcia-Feijoo, $\mathrm{PhD}$ a, b

\footnotetext{
a Ophthalmology Department, Hospital Clínico San Carlos, Instituto de Investigación Sanitaria San Carlos (IdISSC), Madrid, Spain

${ }^{\mathrm{b}}$ Ramon Castroviejo Institute, Universidad Complutense, Madrid, Spain

c Immunology Department, Hospital Clínico San Carlos, Instituto de Investigación Sanitaria San Carlos (IdISSC), Madrid, Spain
} \\ d Ophthalmology Department, Clinica Universitaria de Navarra, Pamplona, Spain
}

\section{A R T I C L E I N F O}

\section{Article history:}

Received 14 December 2016

Received in revised form

21 March 2017

Accepted 21 March 2017

\section{Keywords:}

Cytokine

Glaucoma

Inflammation

Latanoprost

Preservatives

Ocular surface disease

\begin{abstract}
A B S T R A C T
Purpose: To determine variations in cytokine levels of glaucoma patients treated either with preservative-free latanoprost or preserved latanoprost, relative to healthy individuals.

Methods: Tear samples were collected from 39 healthy subjects, 20 glaucoma patients treated with preserved latanoprost, and 20 patients treated with preservative-free latanoprost. A set of 27 inflammatory cytokines was analyzed in each group, including interleukin (IL)-1 $\beta$, IL-1 ra, IL-2, IL-4, IL5, IL-6, IL-7, IL-8, IL9, IL-10, IL-12 (p70), IL-13, IL-15, IL-17, eotaxin, fibroblast growth factor (FGF) basic, granulocyte colony stimulating factor (G-CSF), granulocyte monocyte colony stimulating factor (GM-CSF), interferon (IFN)- $\gamma$, interferon gamma-induced protein (IP)-10, monocyte chemo attractant protein (MCP)-1MCAF, macrophage inflammatory protein (MIP)- $1 \alpha$, MIP-1 $\beta$, platelet-derived growth factor (PDGF)-BB, regulated on activation, normal $T$ cell expressed and secreted (RANTES), tumor necrosis factor (TNF)- $\alpha$ and vascular endothelial growth factor (VEGF). Cytokine concentrations were obtained by the Bio-Plex Human Cytokine Immunoassay. Non-invasive tear breakup time (NI-TBUT), tear meniscus height, corneal fluorescein staining, conjunctival hyperemia and ocular surface disease index (OSDI) were assessed in patients treated with preservative-free and preserved latanoprost.

Results: The levels of IL-2, IL-5, IL-10, IL-12 (p70), IL-13, IL-15, IL-17, FGF basic, PDGF-BB, and TNF- $\alpha$ were significantly higher in patients receiving preserved latanoprost, compared to normal controls $(p<0.05)$. The expression of all the cytokines studied remained statistically invariable in patients receiving preservative-free latanoprost, compared to healthy subjects $(p>0.05)$. Ocular surface parameters were not significantly different in both glaucoma groups, and no correlation between these clinical parameters and cytokine levels was observed.

Conclusions: Treatment with preserved latanoprost has a direct impact on tear cytokine levels, whereas this effect is not observed upon preservative-free latanoprost instillation.
\end{abstract}

(๑) 2017 Elsevier Inc. All rights reserved.

\footnotetext{
Abbreviations: IL, interleukin; FGF, fibroblast growth factor; G-CSF, granulocyte colony stimulating factor; GM-CSF, granulocyte monocyte colony stimulating factor; IFN,

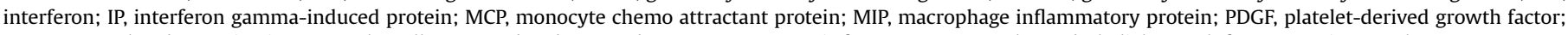

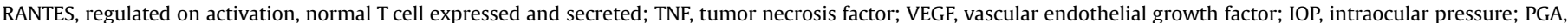

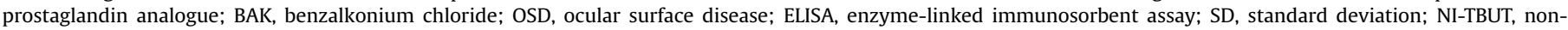
invasive tear breakup time; OSDI, Ocular Surface Disease Index.

* Corresponding author. Hospital Clínico San Carlos, Martin Lagos sn, 28040 Madrid, Spain.

E-mail address: martinezcasa@ya.com (J.M. Martinez-de-la-Casa).
} 


\section{Introduction}

Glaucoma affects over 60 million people worldwide and is the second leading cause of blindness [1]. Elevated intraocular pressure (IOP) is the main risk factor for development of glaucoma. Thus, the goal of the current standard of care is to lower IOP levels. Following this rationale, different topical hypotensive compounds have emerged, including prostaglandin analogues (PGAs), carbonic anhydrase inhibitors, $\beta$-adrenergic receptor antagonists, $\alpha-2$ selective adrenergic agonists, and non-selective adrenergic agonists. Remarkably, their high IOP-reducing efficacy places PGAs as one of the first-line treatments for glaucoma [2].

In PGAs-based formulations, supplementation with preservatives to protect from microbial contamination entails several ocular side effects $[3,4]$. The presence of benzalkonium chloride (BAK) in latanoprost, the most frequently prescribed PGA, is associated with conjunctival hyperemia, superficial punctate keratopathy, or squamous metaplasia [5-7]. Furthermore, long-term exposure to BAK-preserved medications may cause or aggravate pre-existing ocular surface diseases (OSDs), such as dry eye, blepharitis, meibomian gland dysfunction, chronic conjunctival inflammation, or corneal surface impairment [8,9]. These ocular disorders result in eye irritation, blurred vision or tearing, and in a general deterioration of patients' quality of life [10]. To circumvent these detrimental effects, efforts have been made to develop preservative-free formulations for the management of glaucoma. Following this approach, preservative-free latanoprost was developed as a topical glaucoma treatment. Compared with its preserved counterpart, preservative-free latanoprost showed equivalent efficacy in reducing IOP, was better tolerated, and reduced the number of associated ocular adverse events, such as conjunctival hyperemia [11-13]. However, the mechanisms behind these favorable effects are not completely understood.

Inflammation is the key underlying mechanism fueling preservative-associated OSDs. Hypotensive eye drops induce the expression of several cytokines, including interleukins, interferon (IFN)- $\gamma$, and tumor necrosis factor (TNF)- $\alpha[8,14]$. Therefore, cytokines have become reliable biomarkers for the diagnosis, monitoring, and classification of OSDs. Their increasing importance is evidenced by the number of tools applied to detect cytokines in tears, including proteomic techniques, enzyme-linked immunosorbent assay (ELISA), membrane microarrays, and multiplex bead assays [15].

In this study, we compared the cytokine profile of patients treated either with preservative-free latanoprost (Monoprost ${ }^{\circledR}$; Thea, Clermont-Ferrand, France) or preserved latanoprost (Xala$\tan ^{\circledR}$; Pfizer, New York, USA), by using multiplex bead technology. We evaluated changes in the concentration of 27 inflammatory cytokines in glaucoma patients, treated with or without preservatives, relative to healthy patients. To the best of our knowledge, this is the first study comparing the impact in cytokine concentration of long-term instillation with either preserved or preservative-free eye drops.

\section{Material and methods}

\subsection{Participants}

This prospective, observational study was conducted at the Hospital Clínico San Carlos (Madrid, Spain). A total of 79 subjects were prospectively recruited between December 2015 and April 2016. Glaucoma patients were receiving either Monoprost or Xalatan for periods ranging from 6 to 120 months. The study also enrolled 39 healthy age- and sex-matched controls.

The study adhered to the tenets of the Declaration of Helsinki and the Spanish legislation, and was approved by the local Institutional Clinical Research Ethics Committee. Written legallybinding informed consent was obtained from all participants before recruitment.

Exclusion criteria included glaucoma surgery within the last six months, secondary glaucoma (including congenital, traumatic, steroid, uveitic, or angle closure), treatment with concomitant topical medications (such as lubricant eye-drops), any ocular surface disease that could have influenced treatment election, other serious eye disease or syndrome, or any physical or mental problem hindering the realization of tests or examinations required for the study.

Each patient was examined and medical history was recorded in a single visit. The information collected included demographic data, treatment duration, and the type (Monoprost or Xalatan) of eye drops received.

\subsection{Tear collection}

Tear samples were collected using a sterile microcapillary tube from a single eye of each individual. Samples were frozen at $-80^{\circ} \mathrm{C}$ and stored until analysis. To avoid evaporation or degradation, the elapsed time between collection and storage was kept below $15 \mathrm{~min}$. Since tear volumes ranged from 2 to $15 \mu \mathrm{L}$, saline buffer (Braun Mini-Plasco ${ }^{\mathbb{R}}$ ) was added to each sample before storage to reach $50 \mu \mathrm{L}$ (the minimum volume for analysis).

\subsection{Cytokine quantification}

Cytokine concentrations were determined in tear samples using the Bio-Plex Pro Human Cytokine 27-Plex Immunoassay (Bio-Rad Laboratories). This platform is based on fluorescent magnetic beads and allows the simultaneous detection of samples in 96-microwell plates, requiring low sample volumes [16-18]. With this technology, up to 27 relevant inflammatory cytokines could be detected in a single run. The set of cytokines detected were: interleukin (IL)-1 $\beta$, IL-1ra, IL-2, IL-4, IL-5, IL-6, IL-7, IL-8, IL9, IL-10, IL-12 (p70), IL-13, IL15, IL-17, eotaxin, fibroblast growth factor (FGF) basic, granulocyte colony stimulating factor (G-CSF), granulocyte monocyte colony stimulating factor (GM-CSF), interferon (IFN)- $\gamma$, interferon gammainduced protein (IP)-10, monocyte chemo attractant protein (MCP)$1 \mathrm{MCAF}$, macrophage inflammatory protein (MIP)- $1 \alpha$, MIP- $1 \beta$, platelet-derived growth factor (PDGF)-BB, regulated on activation, normal $T$ cell expressed and secreted (RANTES), tumor necrosis factor (TNF)- $\alpha$ and vascular endothelial growth factor (VEGF).

Briefly, $50 \mu \mathrm{L}$ of tear samples were dispensed into defined wells of the 96-microwell plate. Samples were simultaneously read on the Luminex MAGPIX reader (Luminex Corporation, Austin TX USA). Cytokine concentrations were derived by interpolating the measured fluorescence intensities to standard curves, and correcting by the corresponding dilution factor employed to achieve the minimum volume for analysis. Bio-Plex Manager ${ }^{\mathrm{TM}}$ software was employed to calculate cytokine concentrations.

\subsection{Ocular surface measurements}

The ocular surface parameters evaluated included corneal fluorescein staining, non-invasive tear breakup time (NI-TBUT), tear meniscus height, conjunctival hyperemia and ocular surface disease index (OSDI). These parameters were assessed at least $48 \mathrm{~h}$ before or after tear sample acquisition.

NI-TBUT and conjunctival hyperemia were measured by the Keratograph 5 (Oculus, Wetzlar, Germany), and the lower tear meniscus height was evaluated by the Spectralis Fourier-Domain OCT (Heidelberg Engineering GmbH, Germany) [19]. 
All eyes were examined using a standard slit lamp at $\times 16$ magnification. The cornea was stained using fluorescein staining (Minims-fluorescein sodium 2.0\%; Chauvin Pharmaceuticals Ltd.). Corneal staining was graded using the 6 -point Oxford scale $(0=$ no staining; 5 = severe staining). Since all the patients evaluated exhibited a maximum corneal staining score of 1 , this variable was subsequently graded as negative or positive (score 0 or 1 , respectively).

Global OSDI score was calculated for each participant from the following equation: $12.5 \times$ [(sum of individual question scores)/ (number of questions answered)], which yields a global score ranging from 0 to 100 .

\subsection{Statistical methods}

Statistical analyses were performed using SAS software for Windows, version 9.2. To evaluate differences between study groups, quantitative variables were analyzed using the Student's ttest for independent samples, whereas categorical variables were compared using the Chi-square test. Spearman rank correlation was used to analyze the correlations between tear cytokine levels and ocular surface parameters. The adjusted level was established by Bonferroni test. $p<0.05$ was the threshold to determine statistically significant differences.

\section{Results}

\subsection{Demographic characteristics of study groups}

Demographic characteristics of the participants are listed in Table 1. A total of 79 subjects (median age, 70 years; range, 29-88 years) participated in this study; 34 were males (43.0\%) and 45 were females (57.0\%). The study enrolled 40 glaucoma patients, and 39 individuals with no history of ocular disease were enrolled as untreated controls. Among glaucoma patients, 20 were receiving Monoprost and the other 20 Xalatan as topical anti-glaucoma medication. Mean time of treatment did not statistically differ between the two groups $(29.6 \pm 27.2$ months for Monoprost and $33.8 \pm 26.5$ months for Xalatan; $p=0.619$ ). The demographic variables assessed were not significantly different between groups $(p>0.05)$.

\subsection{Effects of Xalatan on tear cytokine levels}

A set of 27 relevant inflammatory cytokines were analyzed in Xalatan-treated and control tears. The absolute concentration of each cytokine is presented in Table 2. Cytokine levels were systematically greater in patients receiving Xalatan, compared to healthy participants (Fig. 1). Among all the cytokines evaluated, there was a significant increase $(p<0.05)$ in the levels of IL-2, IL5, IL-10, IL-12 (p70), IL-13, IL-15, IL-17, FGF basic, PDGF-BB, and TNF- $\alpha$ in Xalatan-treated versus control subjects. Remarkably,
Table 2

Cytokine concentrations in Xalatan-treated and control subjects.

\begin{tabular}{llll}
\hline Cytokine & Control & Xalatan & $p$-value \\
\hline IL-1 $\beta$ & $51.99 \pm 44.38$ & $73.74 \pm 60.01$ & 0.120 \\
IL-1ra & $18,120.10 \pm 17,296.27$ & $14,150.58 \pm 10,067.61$ & 0.359 \\
IL-2 & $79.48 \pm 67.76$ & $130.22 \pm 84.72$ & $0.028^{*}$ \\
IL-4 & $58.93 \pm 40.28$ & $75.10 \pm 53.41$ & 0.198 \\
IL-5 & $150.56 \pm 133.52$ & $258.91 \pm 221.34$ & $0.024^{*}$ \\
IL-6 & $161.79 \pm 150.37$ & $185.92 \pm 131.44$ & 0.546 \\
IL-7 & $442.35 \pm 343.28$ & $631.08 \pm 475.92$ & 0.086 \\
IL-8 & $573.88 \pm 465.33$ & $475.80 \pm 314.45$ & 0.402 \\
IL-9 & $230.65 \pm 168.14$ & $339.96 \pm 244.85$ & 0.050 \\
IL-10 & $243.01 \pm 182.77$ & $392.86 \pm 295.55$ & $0.019^{*}$ \\
IL-12 $(p 70)$ & $334.28 \pm 222.28$ & $505.92 \pm 328.43$ & $0.021^{*}$ \\
IL-13 & $120.99 \pm 95.26$ & $193.62 \pm 129.48$ & $0.017^{*}$ \\
IL-15 & $149.26 \pm 107.10$ & $228.37 \pm 130.37$ & $0.047^{*}$ \\
IL-17 & $343.83 \pm 332.85$ & $634.36 \pm 472.46$ & $0.009^{*}$ \\
Eotaxin & $396.77 \pm 251.72$ & $512.45 \pm 327.51$ & 0.140 \\
FGF basic & $303.67 \pm 215.90$ & $547.02 \pm 355.85$ & $0.002^{*}$ \\
G-CSF & $621.68 \pm 483.07$ & $700.72 \pm 489.66$ & 0.560 \\
GM-CSF & $168.91 \pm 124.44$ & $227.03 \pm 136.41$ & 0.123 \\
IFN- $\gamma$ & $2034.27 \pm 1331.88$ & $2677.13 \pm 1857.30$ & 0.134 \\
IP-10 & $25,632.69 \pm 28,754.16$ & $14,130.95 \pm 15,142.54$ & 0.102 \\
MCP-1 $(M C A F)$ & $489.70 \pm 304.50$ & $633.31 \pm 388.62$ & 0.124 \\
MIP-1 $\alpha$ & $54.92 \pm 38.41$ & $70.14 \pm 44.04$ & 0.176 \\
PDGF-BB & $126.17 \pm 113.44$ & $234.96 \pm 201.35$ & $0.010^{*}$ \\
MIP-1 $\beta$ & $174.03 \pm 176.80$ & $124.14 \pm 77.96$ & 0.249 \\
RANTES & $320.03 \pm 218.74$ & $394.26 \pm 228.71$ & 0.231 \\
TNF- $\alpha$ & $317.12 \pm 263.14$ & $602.44 \pm 502.04$ & 0.006 \\
VEGF & $476.41 \pm 286.34$ & $495.52 \pm 282.81$ & 0.808 \\
\hline
\end{tabular}

Data are expressed as mean $(\mathrm{pg} / \mathrm{mL}) \pm \mathrm{SD}$.

Statistical significance between groups was determined using Student's t-test.

*Indicates a statistically significant difference $(p<0.05)$.

Abbreviations: IL = interleukin; FGF = fibroblast growth factor; G-CSF = granulocyte colony stimulating factor; GM-CSF = granulocyte monocyte colony stimulating factor; IFN = interferon; IP = interferon gamma-induced protein; $\mathrm{MCP} 1(\mathrm{MCAF})=$ monocyte chemo attractant protein; MIP = macrophage inflammatory protein; PDGF = platelet-derived growth factor; RANTES $=$ regulated on activation, normal $\mathrm{T}$ cell expressed and secreted; TNF = tumor necrosis factor; $\mathrm{VEGF}=$ vascular endothelial growth factor; $\mathrm{SD}=$ standard deviation.

PDGF-BB and TNF- $\alpha$ showed the highest increase in concentration (1.9-fold) in patients treated with Xalatan, relative to normal subjects.

\subsection{Effects of Monoprost on tear cytokine levels}

Cytokine concentrations in Monoprost-treated and control tears are given in Table 3. Levels of cytokines were highly coincident for Monoprost and control groups (Fig. 1). Likewise, changes in cytokine concentrations between patients receiving Monoprost and healthy subjects failed to reach statistical significance for all the 27 cytokines studied $(p>0.05)$. Remarkably, those cytokines with significantly increased levels in the previous comparison (Xalatan versus control), remained statistically invariable when Monoprosttreated patients were compared with healthy patients.

Table 1

Demographic characteristics of study participants.

\begin{tabular}{|c|c|c|c|c|c|}
\hline & Control & Monoprost & Xalatan & Total & $p$-value \\
\hline \multicolumn{6}{|l|}{ Gender, N (\%) } \\
\hline Male & $16(41 \%)$ & $10(50 \%)$ & $8(40 \%)$ & $34(43 \%)$ & 0.765 \\
\hline Female & $23(59 \%)$ & $10(50 \%)$ & $12(60 \%)$ & $45(57 \%)$ & \\
\hline Total & 39 (100\%) & $20(100 \%)$ & $20(100 \%)$ & $79(100 \%)$ & \\
\hline Age (years), Mean \pm SD & $72.72 \pm 8.67$ & $70.35 \pm 13.92$ & $71.65 \pm 9.93$ & $71.85 \pm 10.43$ & 0.713 \\
\hline
\end{tabular}

Data are expressed as number (percentage), or mean + SD.

Abbreviations: $\mathrm{N}=$ number of patients; $\mathrm{SD}=$ standard deviation.

Statistical significance between groups was determined using either the Student's t-test or the Chi-square test for continuous or categorical variables, respectively. 


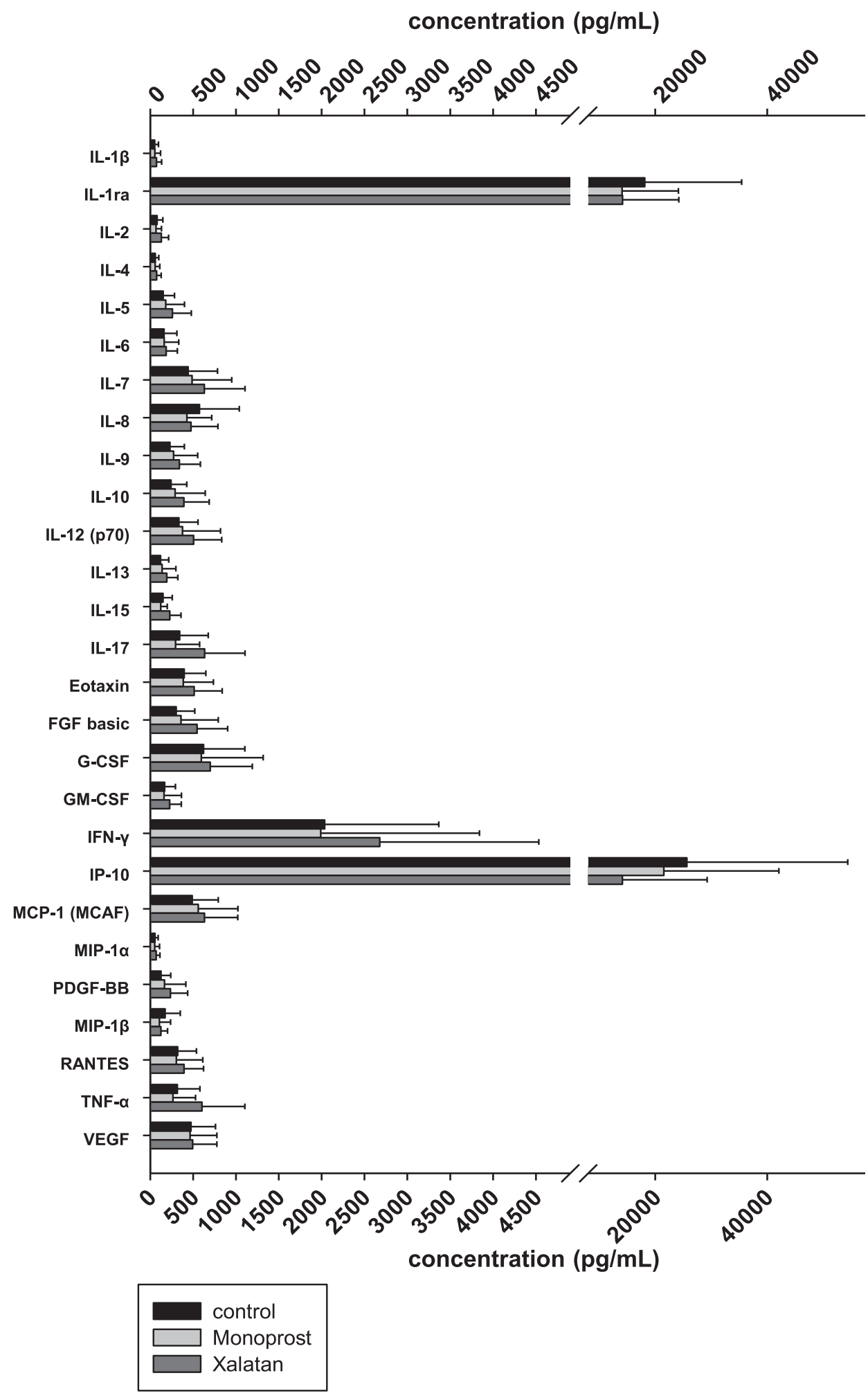

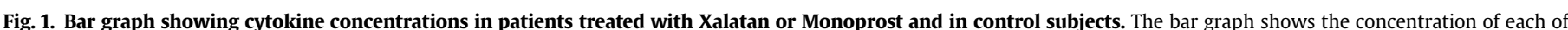

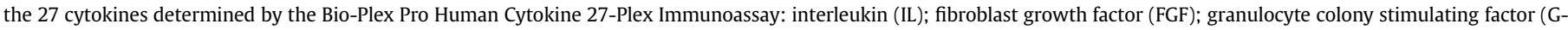

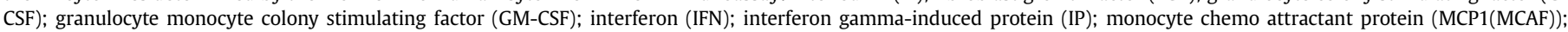

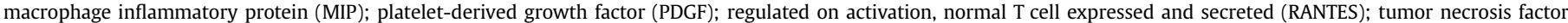
(TNF); vascular endothelial growth factor (VEGF). Values are expressed as mean $(\mathrm{pg} / \mathrm{mL}) \pm$ standard deviation (SD).

\subsection{Cytokine profiles and clinical parameters in Monoprost and} Xalatan groups

The comparison of Xalatan- and Monoprost-treated tears revealed that, except for IP-10, all the cytokines evaluated were higher in the Xalatan group, with the levels of IL-2, IL-15, IL-17 and TNF- $\alpha$ reaching statistical significance $(p<0.05)$ (Table 4 , Fig. 1$)$.

Clinical parameters of ocular surface showed similar levels in 
Table 3

Cytokine concentrations in Monoprost-treated and control subjects.

\begin{tabular}{llll}
\hline Cytokine & Control & Monoprost & $p$-value \\
\hline IL-1 $\beta$ & $53.10 \pm 67.05$ & $51.99 \pm 44.38$ & 0.939 \\
IL-1ra & $14,069.58 \pm 10,054.80$ & $18,120.10 \pm 17,296.27$ & 0.349 \\
IL-2 & $68.69 \pm 63.21$ & $79.48 \pm 67.76$ & 0.592 \\
IL-4 & $57.48 \pm 54.07$ & $58.93 \pm 40.28$ & 0.908 \\
IL-5 & $181.62 \pm 217.64$ & $150.56 \pm 133.52$ & 0.503 \\
IL-6 & $162.51 \pm 171.08$ & $161.79 \pm 150.37$ & 0.987 \\
IL-7 & $488.20 \pm 461.86$ & $442.35 \pm 343.28$ & 0.668 \\
IL-8 & $428.52 \pm 289.48$ & $573.88 \pm 465.33$ & 0.219 \\
IL-9 & $272.26 \pm 281.40$ & $230.65 \pm 168.14$ & 0.492 \\
IL-10 & $290.68 \pm 351.42$ & $243.01 \pm 182.77$ & 0.494 \\
IL-12 $(p 70)$ & $377.24 \pm 443.19$ & $334.28 \pm 222.28$ & 0.620 \\
IL-13 & $138.74 \pm 160.53$ & $120.99 \pm 95.26$ & 0.596 \\
IL-15 & $122.86 \pm 76.12$ & $149.26 \pm 107.10$ & 0.442 \\
IL-17 & $295.49 \pm 280.22$ & $343.83 \pm 332.85$ & 0.604 \\
Eotaxin & $385.54 \pm 352.04$ & $396.77 \pm 251.72$ & 0.889 \\
FGF basic & $358.46 \pm 435.10$ & $303.67 \pm 215.90$ & 0.519 \\
G-CSF & $596.67 \pm 722.30$ & $621.68 \pm 483.07$ & 0.879 \\
GM-CSF & $162.25 \pm 201.80$ & $168.91 \pm 124.44$ & 0.882 \\
IFN- $\gamma$ & $1989.44 \pm 1852.67$ & $2034.27 \pm 1331.88$ & 0.916 \\
IP-10 & $21,529.78 \pm 20,541.95$ & $25,632.69 \pm 28,754.16$ & 0.584 \\
MCP-1 (MCAF) & $560.90 \pm 463.19$ & $489.70 \pm 304.50$ & 0.481 \\
MIP-1 $\alpha$ & $52.88 \pm 55.96$ & $54.92 \pm 38.41$ & 0.870 \\
PDGF-BB & $166.56 \pm 248.59$ & $126.17 \pm 113.44$ & 0.393 \\
MIP-1 $\beta$ & $107.33 \pm 129.72$ & $174.03 \pm 176.80$ & 0.173 \\
RANTES & $305.15 \pm 308.74$ & $320.03 \pm 218.74$ & 0.832 \\
TNF- $\alpha$ & $265.71 \pm 263.83$ & $317.12 \pm 263.14$ & 0.488 \\
VEGF & $464.41 \pm 312.76$ & $476.41 \pm 286.34$ & 0.883 \\
\hline Da & & &
\end{tabular}

Data are expressed as mean $(\mathrm{pg} / \mathrm{mL}) \pm \mathrm{SD}$.

Statistical significance between groups was determined using the Student's t-test Abbreviations: IL = interleukin; FGF = fibroblast growth factor; $\mathrm{G}-\mathrm{CSF}=$ granulocyte colony stimulating factor; GM-CSF = granulocyte monocyte colony stimulating factor; IFN = interferon; IP $=$ interferon gamma-induced protein; $\mathrm{MCP} 1(\mathrm{MCAF})=$ monocyte chemo attractant protein; MIP = macrophage inflammatory protein; PDGF = platelet-derived growth factor; RANTES = regulated on activation, normal $\mathrm{T}$ cell expressed and secreted; TNF = tumor necrosis factor; $\mathrm{VEGF}=$ vascular endothelial growth factor; $\mathrm{SD}=$ standard deviation.

both Monoprost and Xalatan groups. Concretely, tear meniscus height, OSDI, NI-TBUT, and conjunctival hyperemia were statistically equivalent in Monoprost- and Xalatan-treated patients $(p>0.05)$ (Table 5). Corneal fluorescein staining and the presence of epitheliopathy were not significantly different between Monoprost and Xalatan groups $(p=0.491)$.

No significant correlation was observed between cytokine levels and clinical parameters of ocular surface disease (OSDI, NI-TBUT, conjunctival hyperemia, and tear meniscus height), when analyzing those cytokines with significantly different levels in Monoprost and Xalatan groups (IL-2, IL-15, IL-17 and TNF- $\alpha$ ).

\section{Discussion}

Due to the chronic nature of glaucoma, many glaucomatous patients require lifetime treatment. The current goal of antiglaucoma therapy is to lower IOP without compromising patients' quality of life $[10,20]$. The use of PGAs as topical medication entails several adverse events, which often derive from the presence of preservatives in these ophthalmic preparations [21,22]. Adverse events include inflammation or corneal impairment, among other symptoms of discomfort, and can result in treatment discontinuation [23-25]. In this regard, there is increasing concern about the risks associated with the use of preservatives in anti-glaucoma medications.

For this reason, developing new anti-glaucoma formulations without preservatives appears to be a successful therapeutic avenue for the management of glaucoma. Different preservativefree PGAs are currently available that are no less effective in
Table 4

Cytokine concentrations in Monoprost- and Xalatan-treated subjects.

\begin{tabular}{llll}
\hline Cytokine & Monoprost & Xalatan & $p$-value \\
\hline IL-1 $\beta$ & $53.10 \pm 67.05$ & $73.74 \pm 60.01$ & 0.312 \\
IL-1ra & $14,069.58 \pm 10,054,80$ & $14,150,58 \pm 10,067.61$ & 0.980 \\
IL-2 & $68.69 \pm 63.21$ & $130.22 \pm 84.72$ & $0.022^{*}$ \\
IL-4 & $57.48 \pm 54.07$ & $75.10 \pm 53.41$ & 0.307 \\
IL-5 & $181.62 \pm 217.64$ & $258.91 \pm 221.34$ & 0.273 \\
IL-6 & $162.51 \pm 171.08$ & $185.92 \pm 131.44$ & 0.637 \\
IL-7 & $488.20 \pm 461.86$ & $631.08 \pm 475.92$ & 0.341 \\
IL-8 & $428.52 \pm 289.48$ & $475.80 \pm 314.45$ & 0.629 \\
IL-9 & $272.26 \pm 281.40$ & $339.96 \pm 244.85$ & 0.433 \\
IL-10 & $290.68 \pm 351.42$ & $392.86 \pm 295.55$ & 0.326 \\
IL-12 (p70) & $377.24 \pm 443.19$ & $505.92 \pm 328.43$ & 0.303 \\
IL-13 & $138.74 \pm 160.53$ & $193.62 \pm 129.48$ & 0.241 \\
IL-15 & $122.86 \pm 76.12$ & $228.37 \pm 130.37$ & 0.016 \\
IL-17 & $295.49 \pm 280.22$ & $634.36 \pm 472.46$ & $0.014^{*}$ \\
Eotaxin & $385.54 \pm 352.04$ & $512.45 \pm 327.51$ & 0.245 \\
FGF basic & $358.46 \pm 435.10$ & $547.02 \pm 355.85$ & 0.142 \\
G-CSF & $596.67 \pm 722.30$ & $700.72 \pm 489.66$ & 0.603 \\
GM-CSF & $162.25 \pm 201.80$ & $227.03 \pm 136.41$ & 0.267 \\
IFN- $\gamma$ & $1989.44 \pm 1852.67$ & $2677.13 \pm 1857.30$ & 0.248 \\
IP-10 & $21,529.78 \pm 20,541.95$ & $14,130.95 \pm 15,142.54$ & 0.207 \\
MCP-1 (MCAF) & $560.0 \pm 463.19$ & $633.31 \pm 388.62$ & 0.595 \\
MIP-1 $\alpha$ & $52.88 \pm 55.96$ & $70.14 \pm 44.04$ & 0.285 \\
PDGF-BB & $166.56 \pm 248.59$ & $234.96 \pm 201.35$ & 0.345 \\
MIP-1 $\beta$ & $107.33 \pm 129.72$ & $124.14 \pm 77.96$ & 0.636 \\
RANTES & $305.15 \pm 308.74$ & $394.26 \pm 228.71$ & 0.306 \\
TNF- $\alpha$ & $265.71 \pm 263.83$ & $602.44 \pm 502.04$ & $0.013^{*}$ \\
VEGF & $464.41 \pm 312.76$ & $495.52 \pm 282.81$ & 0.743 \\
\hline Da 0 & &
\end{tabular}

Data are expressed as mean $(\mathrm{pg} / \mathrm{mL}) \pm \mathrm{SD}$.

Statistical significance between groups was determined using the Student's t-test. *Indicates a statistically significant difference $(p<0.05)$.

Abbreviations: IL = interleukin; FGF = fibroblast growth factor; G-CSF = granulocyte colony stimulating factor; GM-CSF = granulocyte monocyte colony stimulating factor; IFN = interferon; IP = interferon gamma-induced protein; $\mathrm{MCP} 1(\mathrm{MCAF})=$ monocyte chemo attractant protein; MIP = macrophage inflammatory protein; PDGF = platelet-derived growth factor; RANTES $=$ regulated on activation, normal $\mathrm{T}$ cell expressed and secreted; TNF = tumor necrosis factor; $\mathrm{VEGF}=$ vascular endothelial growth factor; $\mathrm{SD}=$ standard deviation.

Table 5

Ocular surface parameters in Monoprost- and Xalatan-treated patients.

\begin{tabular}{llll}
\hline & Monoprost & Xalatan & $p$-value \\
\hline OSDI & $11.220 \pm 9.799$ & $6.439 \pm 7.052$ & 0.121 \\
Conjunctival Hyperemia & $1.706 \pm 0.522$ & $1.400 \pm 0.473$ & 0.088 \\
Tear Meniscus Height & $324.000 \pm 108.312$ & $333.059 \pm 104.711$ & 0.809 \\
NI-TBUT & $8.836 \pm 4.73,136$ & $11.365 \pm 6.045$ & 0.189 \\
\hline
\end{tabular}

Statistical significance between groups was determined using either the Student's ttest or the Chi-square test for continuous or categorical variables, respectively. Abbreviations: OSDI = ocular surface disease index; NI-TBUT $=$ non-invasive tear breakup time.

reducing IOP than their preserved counterparts [26-28]. Furthermore, switching studies revealed that changing from preserved to preservative-free medications significantly improved signs and symptoms related to OSDs [29,30].

Among PGAs, we focused on latanoprost, the most frequently prescribed formulation, because of its high tolerability and the lesser number of side effects associated with its use [7]. Despite its potency, various adverse events have been associated with the presence of BAK in latanoprost formulation [5-7]. Preservative-free latanoprost (Monoprost) was therefore conceived to maintain the drug potency of latanoprost while reducing the associated adverse events.

A variety of studies support the advantages of Monoprost as topical anti-glaucoma medication. In an in vivo study, Pauly and colleagues revealed that epithelial cell viability was higher after treatment with preservative-free latanoprost compared to 
treatment with preserved latanoprost [11]. A randomized, multinational trial demonstrated equivalent efficacy between preserved and preservative-free latanoprost in reducing IOP levels [12]. A meta-analysis of randomized clinical trials confirmed that preservative-free latanoprost presented equivalent safety and reduced adverse events, such as conjunctival hyperemia, compared to preserved latanoprost [31]. A phase 2 study reported no differences between latanoprost with or without preservative, in terms of safety and patient tolerance [32]. Additionally, commercialization in single-dose format ensures Monoprost sterility.

Several studies have revealed important differences between preserved and preservative-free latanoprost, but none has directly compared their impact on tear cytokine levels. Inflammation is a key cause and effect of the onset and progression of OSDs, which can be reliably monitored by measuring cytokine levels. In this study, we compared differences in cytokine concentrations promoted either by Monoprost or Xalatan in glaucoma patients. We observed that treatment with Xalatan resulted in great variations of cytokine levels in tears. However, and interestingly, upon treatment with Monoprost, we only observed mild changes that did not reach statistical significance.

We simultaneously determined the concentration of 27 cytokines employing multiplex technology, thus eliminating inter-assay variability. The cytokine profile of patients treated with Xalatan revealed a significant increase in IL-2, IL-5, IL-10, IL-12 (p70), IL-13, IL-15, IL-17, FGF basic, PDFG-BB, and TNF- $\alpha$. Among them, PDFG-BB and TNF- $\alpha$ showed the highest increase in concentration (1.9-fold) in patients treated with Xalatan, whereas their expression was statistically unchanged in patients treated with Monoprost. TNF- $\alpha$ is a pleiotropic protein eliciting different cellular responses, such as apoptotic cell death or inflammation [33]. Consistently, both apoptosis and inflammation participate in ocular adverse events associated with the use of preservatives in ophthalmic preparations. Indeed, BAK is able to interact with cell death receptors, inducing apoptosis and inflammation [34,35]. This fact, together with the elevated levels of TNF- $\alpha$ in patients treated with Xalatan, suggests that apoptosis could also play a role in these associated side effects. In addition to playing this role, TNF- $\alpha$ is a recognized marker of dry eye disease $[18,36]$. In line with this, growth factors such as PDGF-BB and FGF basic play a critical role in the maintenance of corneal function and imbalances in their expression are found in different ocular pathologies [37,38]. In addition, IL-17 (the IL presenting the highest increase) is also associated with the severity of different OSDs, such as uveitis, scleritis and dry eye disease [39-41]. Overall, patients treated with Xalatan presented an increased expression of different OSD markers, which could indicate that they are more prone to suffer or exacerbate these ophthalmic pathologies.

Nevertheless, other relevant markers of OSDs, such as IL- $\beta 1$ or IL-6, are not differentially expressed in patients treated with Xalatan [42]. This apparent discrepancy could be explained considering that early and late inflammatory responses greatly differ in their cytokine profile. In this regard, this study was conducted in individuals already under treatment for long-term periods (ranging from 6 to 120 months). This fact represents an important strength of our study, since it reflects the actual context of patients chronically exposed to anti-glaucoma treatments. In contrast, clinical trials are usually performed according to shortterm schedules, thus underestimating potential long-term effects. Unexpectedly, no relevant differences in ocular surface measurements (corneal staining, conjunctival hyperemia, NI-TBUT, and tear meniscus height) were observed between Xalatan- and Monoprosttreated patients. In addition, cytokine levels and these ocular surface parameters were not statistically correlated in either the Xalatan or the Monoprost groups. Thus, although the presence of
BAK is not apparently influencing these ocular surface markers, it could be impacting other clinical outcomes, such as the success of posterior glaucoma surgery. The lack of correlation indicates that the inflammatory status achieved after long-term treatment with either Monoprost or Xalatan is not directly translated in detectable differences by exploratory procedures. This fact could be explained considering the wide variation of treatment durations that could compensate potential differences in clinical measurements between both groups. This idea is in accordance with previous studies in which transition from latanoprost to BAK-free tavoprost showed that hyperemia was not significantly altered at 1 month, but it was significantly decreased after 3-12 months of treatment [43]. Hence, it would be interesting to unveil the inflammatory profile of patients as a function of treatment duration, to distinguish between acute and chronic adverse events derived from the use of preservatives, and to establish its potential correlation with ocular surface markers.

A limitation of this study is that basal levels of cytokines were not determined before treatment initiation. However, since patient selection was not based on clinical characteristics or previous treatment intolerances, we can assume that cytokine levels were balanced between both groups. Further studies should be conducted using a crossover design switching from preserved to preservative-free latanoprost, and vice versa. By quantifying cytokine levels before and after each treatment course, we could rule out any baseline effect.

Taken together, the results of this study support previous reports of the beneficial effects of Monoprost in patients, and highlights the importance of assessing the cytokine profile to characterize inflammatory effects of topical medications.

\section{Conclusions}

This study compared the cytokine profile of patients treated with preservative-free or preserved latanoprost. The lack of inflammation associated with preservative-free latanoprost treatment is likely responsible for its significant reduction of adverse events. These findings may have special relevance for patients receiving long-term treatment, and with OSDs.

\section{Disclosure}

This work was supported by Laboratios Thea. Laboratios Thea contributed to cytokine detection test and manuscript preparation.

The authors have no commercial or proprietary interest in any concept or product described in this article.

\section{Acknowledgments}

We would like to thank Red temática de Investigación Cooperativa Oftared, Enfermedades oculares: Prevención, detección precoz y tratamiento de la patología ocular prevalente degenerativa y crónica (Instituto de Salud Carlos III), and Grupo de Investigación 920415-GR58/08 (Universidad Complutense de Madrid).

\section{References}

[1] Quigley HA, Broman AT. The number of people with glaucoma worldwide in 2010 and 2020. Br J Ophthalmol 2006;90:262-7. http://dx.doi.org/10.1136/ bjo.2005.081224.

[2] Kanner E, Tsai JC. Glaucoma medications: use and safety in the elderly population. Drugs Aging 2006;23:321-32. http://dx.doi.org/10.2165/00002512200623040-00005.

[3] Jaenen N, Baudouin C, Pouliquen P, Manni G, Figueiredo A, Zeyen T. Ocular symptoms and signs with preserved and preservative-free glaucoma medications. Eur J Ophthalmol 2007;17:341-9.

[4] Bagnis A, Papadia M, Scotto R, Traverso CE. Antiglaucoma drugs: the role of 
preservative-free formulations. Saudi J Ophthalmol Off J Saudi Ophthalmol Soc 2011;25:389-94. http://dx.doi.org/10.1016/j.sjopt.2011.08.004.

[5] Ishida N, Odani-Kawabata N, Shimazaki A, Hara H. Prostanoids in the therapy of glaucoma. Cardiovasc Drug Rev 2006;24:1-10. http://dx.doi.org/10.1111/ j.1527-3466.2006.00001.x.

[6] Baudouin C, Labbé A, Liang H, Pauly A, Brignole-Baudouin F. Preservatives in eyedrops: the good, the bad and the ugly. Prog Retin Eye Res 2010;29: 312-34. http://dx.doi.org/10.1016/j.preteyeres.2010.03.001.

[7] Orme M, Collins S, Loftus J. Long-term medical management of primary openangle glaucoma and ocular hypertension in the UK: optimizing costeffectiveness and clinic resources by minimizing therapy switches. J Glaucoma 2012;21:433-49. http://dx.doi.org/10.1097/ IJG 0b013e31821dac2a.

[8] Baudouin C, Liang H, Hamard P, Riancho L, Creuzot-Garcher C, Warnet J-M, et al. The ocular surface of glaucoma patients treated over the long term expresses inflammatory markers related to both T-helper 1 and T-helper 2 pathways. Ophthalmology 2008;115:109-15. http://dx.doi.org/10.1016 j.ophtha.2007.01.036.

[9] Arita R, Itoh K, Maeda S, Maeda K, Furuta A, Tomidokoro A, et al. Effects of long-term topical anti-glaucoma medications on meibomian glands. Graefes Arch Clin Exp Ophthalmol 2012;250:1181-5. http://dx.doi.org/10.1007/ s00417-012-1943-6.

[10] Skalicky SE, Goldberg I, McCluskey P. Ocular surface disease and quality of life in patients with glaucoma. Am J Ophthalmol 2012;153:1-9. http://dx.doi.org 10.1016/j.ajo.2011.05.033. e2.

[11] Pauly A, Roubeix C, Liang H, Brignole-Baudouin F, Baudouin C. In vitro and in vivo comparative toxicological study of a new preservative-free latanoprost formulation. Invest Ophthalmol Vis Sci 2012;53:8172-80. http://dx.doi.org/ 10.1167/iovs.12-10766.

[12] Rouland J-F, Traverso CE, Stalmans I, Fekih L El, Delval L, Renault D, et al. Efficacy and safety of preservative-free latanoprost eyedrops, compared with BAK-preserved latanoprost in patients with ocular hypertension or glaucoma. Br J Ophthalmol 2013;97:196-200. http://dx.doi.org/10.1136/bjophthalmol2012-302121.

[13] Sanford M. Preservative-free latanoprost eye drops in patients with primary open-angle glaucoma/ocular hypertension. Clin Drug Invest 2014;34:521-8. http://dx.doi.org/10.1007/s40261-014-0203-4.

[14] De Paiva CS, Villarreal AL, Corrales RM, Rahman HT, Chang VY, Farley WJ, et al. Dry eye-induced conjunctival epithelial squamous metaplasia is modulated by interferon-gamma. Invest Ophthalmol Vis Sci 2007;48:2553-60. http:// dx.doi.org/10.1167/iovs.07-0069.

[15] Wei Y, Gadaria-Rathod N, Epstein S, Asbell P. Tear cytokine profile as a noninvasive biomarker of inflammation for ocular surface diseases: standard operating procedures. Invest Ophthalmol Vis Sci 2013;54:8327-36. http:// dx.doi.org/10.1167/iovs.13-12132.

[16] Chong RS, Jiang YZ, Boey PY, Yu SJ, Htoon HM, Aung T, et al. Tear cytokine profile in medicated glaucoma patients. Ophthalmology 2010;117:2353-8. http://dx.doi.org/10.1016/j.ophtha.2010.03.064.

[17] Hagan S, Tomlinson A. Tear fluid biomarker profiling: a review of multiplex bead analysis. Ocul Surf 2013;11:219-35. http://dx.doi.org/10.1016/ j.jtos.2013.04.004.

[18] Willcox MDP, Zhao Z, Naduvilath T, Lazon de la Jara P. Cytokine changes in tears and relationship to contact lens discomfort. Mol Vis 2015;21:293-305.

[19] Arriola-Villalobos P, Fernández-Vigo JI, Díaz-Valle D, Peraza-Nieves JE, Fernández-Pérez C, Benítez-Del-Castillo JM. Assessment of lower tear meniscus measurements obtained with keratograph and agreement with Fourier-domain optical-coherence tomography. Br J Ophthalmol 2015;99: 1120-5. http://dx.doi.org/10.1136/bjophthalmol-2014-306453.

[20] Bron AJ, Tomlinson A, Foulks GN, Pepose JS, Baudouin C, Geerling G, et al. Rethinking dry eye disease: a perspective on clinical implications. Ocul Surf 2014;12:S1-31. http://dx.doi.org/10.1016/j.jtos.2014.02.002.

[21] Baudouin C. Detrimental effect of preservatives in eyedrops: implications for the treatment of glaucoma. Acta Ophthalmol 2008;86:716-26. http:// dx.doi.org/10.1111/j.1755-3768.2008.01250.x.

[22] Leung EW, Medeiros F, Weinreb RN. Prevalence of ocular surface disease in glaucoma patients. J Glaucoma 2008;17:350-5. http://dx.doi.org/10.1097/ IJG.0b013e31815c5f4f.

[23] Furrer P, Mayer JM, Gurny R. Ocular tolerance of preservatives and alternatives. Eur J Pharm Biopharm 2002;53:263-80.

[24] Arias A, Schargel K, Ussa F, Canut MI, Robles A y B, Sánchez BM. Patient persistence with first-line antiglaucomatous monotherapy. Clin Ophthalmol 2010;4:261-7. http://dx.doi.org/10.2147/OPTH.S7971.

[25] Fechtner RD, Godfrey DG, Budenz D, Stewart JA, Stewart WC, Jasek MC. Prevalence of ocular surface complaints in patients with glaucoma using topical intraocular pressure-lowering medications. Cornea 2010;29:618-21. http://dx.doi.org/10.1097/ICO.0b013e3181c325b2.

[26] Pisella PJ, Pouliquen P, Baudouin C. Prevalence of ocular symptoms and signs with preserved and preservative free glaucoma medication. $\mathrm{Br} \mathrm{J}$ Ophthalmol 2002;86:418-23.

[27] Esaki Y, Shimazaki A, Pellinen P. Ocular tolerability of preservative-free tafluprost and latanoprost: and comparative study. Open Ophthalmol J 2016;10:146-53. http://dx.doi.org/10.2174/1874364101610010146.

[28] Takagi Y, Osaki H, Yamashita T, Kai Y. Prospective observational postmarketing study of tafluprost $0.0015 \% /$ timolol $0.5 \%$ combination ophthalmic solution for glaucoma and ocular hypertension: short-term efficacy and safety. Ophthalmol Ther 2016. http://dx.doi.org/10.1007/s40123-016-0057-3.

[29] Henry JC, Peace JH, Stewart JA, Stewart WC. Efficacy, safety, and improved tolerability of travoprost BAK-free ophthalmic solution compared with prior prostaglandin therapy. Clin Ophthalmol 2008;2:613-21. http://dx.doi.org/ 10.2147/OPTH.S3881.

[30] Keating GM. Tafluprost Ophthalmic solution $0.0015 \%$ : a review in glaucoma and ocular hypertension. Clin Drug Invest 2016;36:499-508. http:// dx.doi.org/10.1007/s40261-016-0413-z.

[31] Cucherat M, Stalmans I, Rouland J-F. Relative efficacy and safety of preservative-free latanoprost ( $\mathrm{t} 2345)$ for the treatment of open-angle glaucoma and ocular hypertension. J Glaucoma 2014;23:e69-75. http:/ dx.doi.org/10.1097/IJG.0b013e3182a075e6.

[32] Aptel F, Choudhry R, Stalmans I. Preservative-free versus preserved latanoprost eye drops in patients with open-angle glaucoma or ocular hypertension. Curr Med Res Opin 2016;32:1457-63. http://dx.doi.org/10.1080/ 03007995.2016.1202818

[33] Wajant H, Pfizenmaier K, Scheurich P. Tumor necrosis factor signaling. Cell Death Differ 2003;10:45-65. http://dx.doi.org/10.1038/sj.cdd.4401189.

[34] Buron N, Micheau O, Cathelin S, Lafontaine P-O, Creuzot-Garcher C, Solary E. Differential mechanisms of conjunctival cell death induction by ultraviolet irradiation and benzalkonium chloride. Invest Ophthalmol Vis Sci 2006;47: 4221-30. http://dx.doi.org/10.1167/iovs.05-1460.

[35] Aragona P, Aguennouz M, Rania L, Postorino E, Sommario MS, Roszkowska AM, et al. Matrix metalloproteinase 9 and transglutaminase 2 expression at the ocular surface in patients with different forms of dry eye disease. Ophthalmology 2015;122:62-71. http://dx.doi.org/10.1016/ j.ophtha.2014.07.048.

[36] Luo L, Li D-Q Doshi A, Farley W, Corrales RM, Pflugfelder SC. Experimental dry eye stimulates production of inflammatory cytokines and MMP-9 and activates MAPK signaling pathways on the ocular surface. Invest Ophthalmol Vis Sci 2004;45:4293-301. http://dx.doi.org/10.1167/iovs.03-1145.

[37] Imanishi J, Kamiyama K, Iguchi I, Kita M, Sotozono C, Kinoshita S. Growth factors: importance in wound healing and maintenance of transparency of the cornea. Prog Retin Eye Res 2000;19:113-29. http://dx.doi.org/10.1016/ S1350-9462(99)00007-5.

[38] Bradley JC, Bradley RH, McCartney DL, Mannis MJ. Serum growth factor analysis in dry eye syndrome. Clin Exp Ophthalmol 2008;36:717-20. http:// dx.doi.org/10.1111/j.1442-9071.2008.01895.x.

[39] Na K-S, Mok J-W, Kim JY, Rho CR, Joo C-K. Correlations between tear cytokines, chemokines, and soluble receptors and clinical severity of dry eye disease. Invest Ophthalmol Vis Sci 2012;53:5443-50. http://dx.doi.org/ 10.1167/iovs.11-9417.

[40] Kang MH, Kim MK, Lee HJ, Lee H Il, Wee WR, Lee JH. Interleukin-17 in various ocular surface inflammatory diseases. J Korean Med Sci 2011;26:938-44. http://dx.doi.org/10.3346/jkms.2011.26.7.938.

[41] Tan X, Sun S, Liu Y, Zhu T, Wang K, Ren T, et al. Analysis of Th17-associated cytokines in tears of patients with dry eye syndrome. Eye 2014;28:608-13. http://dx.doi.org/10.1038/eye.2014.38.

[42] Massingale ML, Li X, Vallabhajosyula M, Chen D, Wei Y, Asbell PA. Analysis of Inflammatory cytokines in the tears of dry eye patients. Cornea 2009;28: 1023-7. http://dx.doi.org/10.1097/ICO.0b013e3181a16578.

[43] Aihara M, Otani S, Kozaki J, Unoki K, Takeuchi M, Minami K, et al. Long-term effect of bak-free travoprost on ocular surface and intraocular pressure in glaucoma patients after transition from latanoprost. J Glaucoma 2012;21: 60-4. http://dx.doi.org/10.1097/IJG.0b013e3181fc8129. 